\title{
Efficient Classification using Average Weighted Pattern Score with Attribute Rank based Feature Selection
}

\author{
S. Sathya Bama \\ Independent Researcher, Lawley Road, Coimbatore, India - 641003 \\ E-mail: ssathya21@gmail.com \\ A. Saravanan \\ Department of MCA, Sree Saraswathi Thyagaraja College, Tamil Nadu, India - 642107 \\ Corresponding Author E-mail: a.saravanan21@gmail.com
}

Received: 09 January 2019; Accepted: 19 March 2019; Published: 08 July 2019

\begin{abstract}
Classification is found to be an important field of research for many applications such as medical diagnosis, credit risk and fraud analysis, customer segregation, and business modeling. The main intention of classification is to predict the class labels for the unlabeled test samples using a labelled training set accurately. Several classification algorithms exist to classify the test samples based on the trained samples. However, they are not suitable for many real world applications since even a small performance degradation of classification algorithms may lead to substantial loss and crucial implications. In this paper, a simple classification method using the average weighted pattern score with attribute rank based feature selection has been proposed. Feature selection is carried out by computing the attribute score based ranking and the classification is performed using average weighted pattern computation. Experiments have been performed with 40 standard datasets and the results are compared with other classifiers. The outcome of the analysis shows the good performance of the proposed method with higher classification accuracy.
\end{abstract}

Index Terms-Classification, outlier detection, pattern matching, feature selection, attribute rank score.

\section{INTRODUCTION}

Due to the technological improvement and growth of emerging technologies, the storage of data in all fields is getting multiplied for every microsecond. This explosive growth of collected data has to be analyzed for extracting valuable information and to transform them into interesting knowledge. Classification and predictions are the two main areas that are focused on almost all the fields for improving the trends, making decisions and predicting future events based on the collected data. Data mining and machine learning algorithms, generally build a mathematical model on the training sample dataset, in order to make forecasts or decisions by analyzing the data. Data mining is the process of discovering patterns in large data sets from various applications involving methods at the intersection of machine learning, statistics, and database systems [1]. Data Mining is the technical base for Machine learning (ML) which is the study of algorithms and mathematical models used to improve the performance of the applications [2].

Supervised learning is the primary context in data mining and machine learning fields. In general, supervised learning creates and trains a model to predict the future based on the results from the analysis of past history. More technically, supervised learning algorithms have a set of training samples each of which has the input object (set of attributes) and output object (class labels). Once the unlabeled test samples are given as input to the supervised learning algorithm, it examines the characteristics and class labels of training samples and based on which it predicts the class labels for the unlabeled input test samples [3]. Thus, categorizing, classifying or predicting the class labels for unlabeled test samples with the help of a trained model is known as classification. Several classification algorithms are generally available in data mining and machine learning context.

In the classification problem, feature selection is an important task which includes selecting the relevant feature for constructing the model as the dataset contains some features that are redundant and irrelevant features. These irrelevant features that are not interesting for the study can be removed during the classification process [4]. Some of the classification algorithms that are used widely in various applications are eager learners such as Artificial neural networks [5], decision trees [6], naïve bayes [7], instance based classifiers [8] such as nearest neighbour [9], statistical models such as linear regression [10], logistic regression [11], nature-inspired techniques such as genetic programming [12] and ensemble based learners [13] such as random forest [14], adaboost [15], and support vector machine [16]. Though several 
methods exist to classify the dataset, they suffer from several issues. Simple methods generally provide low classification accuracy. Many difficult methods even provide misclassification. Pretentious and complex methods that are having high prediction rate, however, suffer from computational complexity.

This paper presents an instance based classifier algorithm with an average weighted pattern score with attribute rank based feature selection to predict the class labels for the unlabeled test samples. This method is simple however, it produces better classification accuracy than many other traditional methods. An attribute score based ranking algorithm has been proposed to select the relevant attributes by calculating the attribute rank by taking the number of unique values in the set of training samples into account. The attribute rank is converted to attribute weight using the rank sum weight method. The attributes of the test sample are compared with the attributes of the training samples to find the matched pattern. The pattern score of the test sample for each training sample is calculated using the attribute weight and are grouped based on the class label. The average pattern score for each group is calculated and the class group having a maximum score is predicted as the class label for the unlabeled training sample. The experimental study has been performed with 40 standard datasets available at UCI [17] and KEEL [18] repository. The proposed algorithm is compared with other existing methods and the classification accuracy and AUC values are evaluated and analyzed using statistical tests. Through the analysis, the proposed system provides better results than other algorithms.

The organization of the paper is as follows. Section II presents the related work from the literature survey. Section III explains the proposed methodology in details. Experimental analysis is presented in section IV with experimental setup and result analysis. Finally, the paper concludes the work with a conclusion.

\section{RELATED WORKS}

Several methods and their variations exist in the literature for classifying the unlabeled samples. Eager learners generally create a learning model based on the training data and classify the unlabeled sample based on the created model. An artificial neural network is an idea inspired by biological neural networks with a system of interconnected 'neurons' calculating values from the input. Even if the implementation of the model is simple, the processing time increases with the increase in the neural network and are prone to overfitting [19]. Decision trees are tree based predictive model that identifies the target class for the given sample by partitioning the trained samples. The main drawback of decision trees is that in some cases the splitting process may lead to loss of information [20]. Another common eager learner is naïve bayes classifier. It is a probabilistic predictive model that applies Bayes theorem for predicting the class label. However, the accuracy of the prediction decreases with the decrease in the number of training samples [21].
Given a set of training sample with attributes having numeric values, Linear regression fits a linear function to a set of input-output pairs. [22]. Generally, the model is limited to the linear relationship between a set of dependent and independent variables. Similar to linear regression, Logistic regression can be employed for the binary/multivariate classification task. However, the model works well only if the number of output variables is minimum [23]. The main idea of ensemble-based learners is to convert weak learners to strong learners by applying several homogeneous or heterogeneous classifier model. Random forest classifier usually builds multiple random trees by selecting a random sample with replacement and by selecting random attributes from the training set for classifying the unlabeled test data using majority votes [24]. However, the computational complexity and time to build the random trees are really high. Boosting is a method that involves combining heterogeneous learning algorithms for improving the performance of the system. Adaptive Boosting (AdaBoost) classifier combines several heterogeneous weak classifiers to make a strong classifier. AdaBoost provides better accuracy in classifying data samples but is prone to overfitting [25].

Support vector machine is a good choice for various classification problems. However, choosing the best kernel suitable for the application is difficult. Also, they are prone to noise and missing values [16-26]. Instance based classifiers are the most widely used classification as it is simple and easy to understand. K-nearest neighbor classifier is the simple classification model where the input consists of $\mathrm{k}$ nearest training samples and the class label for the test sample is predicted by a majority vote of its neighbors [27]. A new variation of $\mathrm{kNN}$ method based on a two-sided mode, called general nearest neighbor (GNN) rule has been suggested. But the main disadvantage of these methods is that they rigorously deteriorate with noisy data or high dimensionality and the performance becomes very slow [28]. In reference [29], Peng et al. (2009) proposed a new instance based classifier named as Data Gravitation based Classification (DGC) [30,31]. This algorithm basically classifies the test samples by comparing the data gravitation between the different data classes. In reference [32], Cano et al. (2013) extended the DGC by improving the classification accuracy and is called Weighted Data Gravitation based Classification (DGC+) but with the highest complexity.

\section{AVERAge Weighted Pattern Score With ATTRIBUTE RANK BASED FEATURE SELECTION}

The proposed classification method uses average weighted pattern score along with attribute rank for feature selection. The PMC algorithm proposed by the authors Sreeja and Sankar [33] has been taken as a base and further extended by introducing the weights and rank weights for the attributes in the process of feature selection. The overall architecture of the proposed methodology is given in Fig.1.

The proposed method uses the attribute scoring based 
feature selection with the weighted pattern matching mechanism. Consider the dataset $D$ consisting of $n$ attributes denoted by $a_{1}, a_{2}, a_{3}, \ldots, a_{n}$ and $m$ instance denoted by $i_{1}, i_{2}, i_{3}, \ldots, i_{m}$. It is depicted in the following matrix form in (1).

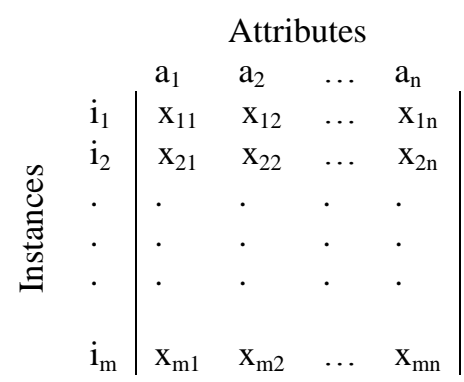

In the above matrix $\mathrm{x}_{\mathrm{m} 1}, \mathrm{x}_{\mathrm{m} 2}, \ldots, \mathrm{x}_{\mathrm{mn}}$ are the values of the $n$ attributes of the $m^{\text {th }}$ instance. Specifically, each instance belongs to a class $C_{k}$ where $k$ takes the values 1 , $2, \ldots, p$.

As a pre-processing step, the prominence of the attribute in the classification process is calculated. All the attributes are ranked based on their importance. Let $\mathrm{R}$ be the ranker function that assigns a value to each attribute $a_{j}$ that belongs to the dataset $\mathrm{D}$ based on the relevance score. It then returns the list of attributes well-arranged based on their relevancy and the formula is

$$
R\left(a_{1}, a_{2}, a_{3}, \ldots a_{n}\right)=<a_{1}^{\prime}, a_{2}^{\prime}, a_{3}^{\prime} \ldots a_{n}^{\prime}>.
$$

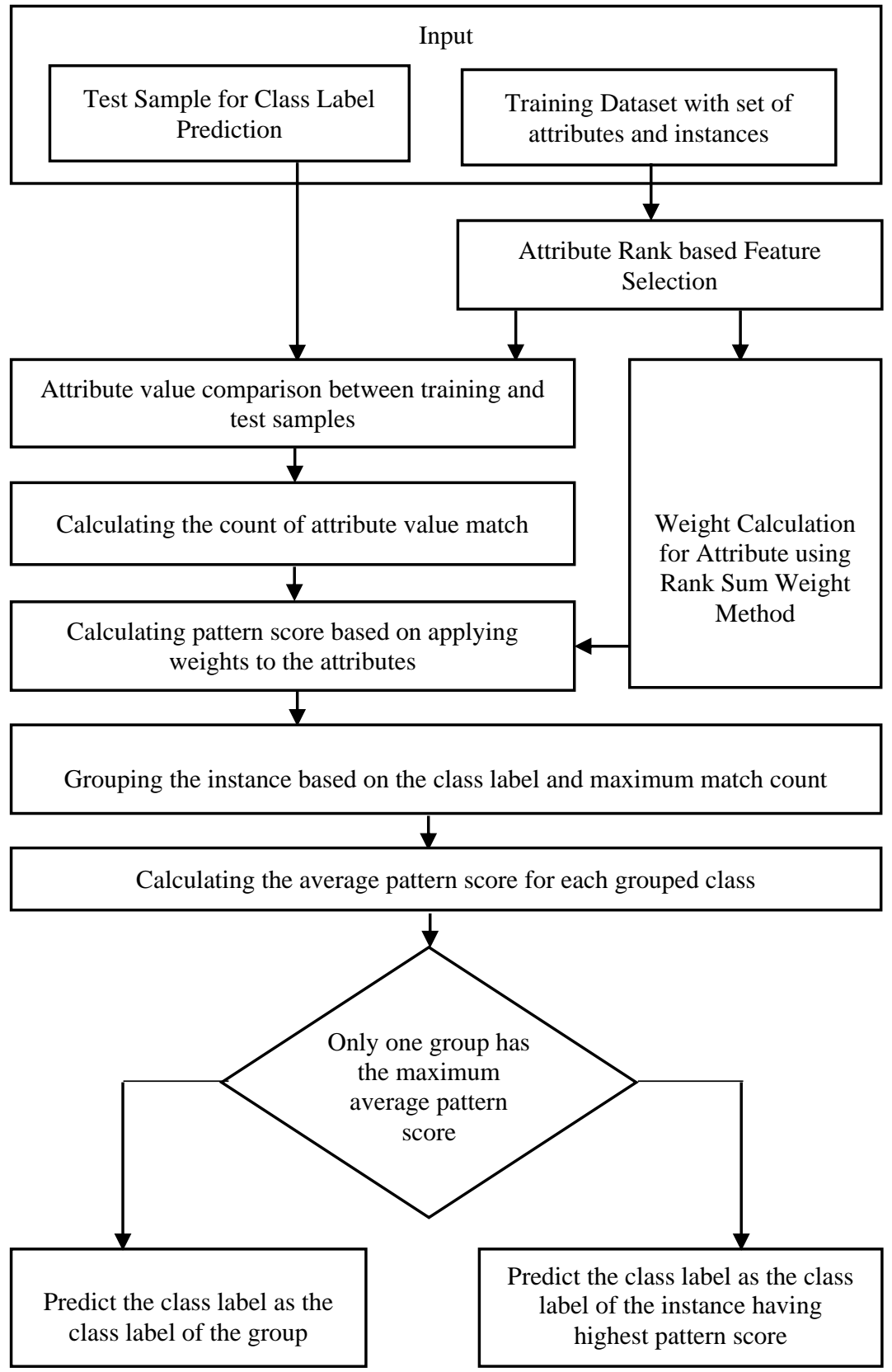

Fig.1. Architecture of the proposed classification method. 
Here, the list of attributes $a_{1}, a_{2}, a_{3}, \ldots, a_{n}$ take the ranks $1,2, \ldots, \mathrm{n}$. The attributes that are not relevant can be removed based on the relevance score. Only the top relevant attributes having the highest score is selected. Let $\mathrm{q}$ be the number of attributes selected as relevant for the classification.

Each selected ranked attribute $a_{1}^{\prime}, a_{2}{ }^{\prime}, a_{3}, \ldots, a_{q}$ is then assigned a weight based on the rank sum weight method. By using the rank sum weight method, the weights are calculated and are normalized in such a way that the sum of weights of the ranked attributes is 1 and the formula is depicted in (3).

$$
w\left(a_{j}\right)=\frac{\left(n-r\left(a_{j}\right)+1\right)}{\sum_{i=1}^{q}\left(n-r\left(a_{i}\right)+1\right)}
$$

The main aim of the proposed classifier is to identify the class label for the unlabeled test instances. The proposed method uses PMC [33] to find the instances with the highest attribute match count by comparing the selected attribute values of the training instances and the test instances as shown in (4) and (5). The matched count of $\mathrm{m}^{\text {th }}$ instance is represented as $\mathrm{n}_{\mathrm{n}}(\mathrm{m})$ and the calculation is given in (4).

$$
n_{a^{\prime}}(m)=\sum_{i=1}^{q} s\left(a_{i}\right)
$$

where $s\left(a_{i}\right)$ is the attribute match score which takes the value either 0 or 1 . If the value of attribute $a_{i}$ of the test sample equals the corresponding attribute value of the training sample then the score will be 1 else the score will be 0 . This can be represented as in (5).

$$
s\left(a_{i}\right)=\left\{\begin{array}{c}
1, \text { if the value of } a_{i} \text { matches } \\
\quad \text { with the training sample } a_{i m} \\
0, \quad \text { Otherwise }
\end{array}\right.
$$

The method then selects the training samples having the highest attribute match count with the given test sample. The selected training instances are grouped based on the class labels as shown in (6) [33].

$$
i_{k} \in \mathbb{G}_{c} \text { iff } n_{a^{\prime}}(k)=\text { maximum and } i_{k} \in C_{j}
$$

c in $\mathbb{G}_{c}$ represents the number of classes in the selected training set samples. All instances having maximum $n_{a^{\prime}}$ and belonging to the class $C_{j}$ is grouped. The rank weights of the selected attributes are applied to the selected training instances to find the pattern score of the test sample $\mathrm{t}$ with respect to the training samples $\mathrm{x}_{\mathrm{i}}$ which is represented as $P S\left(x_{i}\right)$ and the formula is depicted as

$$
P S\left(x_{i}\right)=\sum_{i=1}^{q} s\left(a_{i}\right) * w\left(a_{i}\right)
$$

The average pattern score $P S\left(x_{i c}\right)$ of class $\mathrm{c}$ is calculated by finding the average score of each group in the selected training set samples. The pattern score of the test sample $P S_{\text {test }}$ is the maximum score found among the averaged pattern score of groups $\mathbb{G}_{c}$. Finally, the class label for the test sample is predicted as the class group having a maximum average pattern score and the details are represented in (8) and (9).

$$
\begin{gathered}
P S_{\text {test }}=\max \left(P S\left(x_{c}\right)\right) \\
i_{\text {test }} \in C_{j} \text { iff } P S\left(x_{c}\right)=P S_{\text {test }}
\end{gathered}
$$

If more than one group arrive the maximum average pattern score, then the class label is predicted using the individual instance pattern score. The class label for the test sample is predicted as the class label of the training instance having maximum pattern score since the topmost attribute that is relevant is highly involved in determining the class label and expression is shown in (10).

$$
i_{\text {test }} \in \operatorname{class}(\max (P S(x))
$$

The algorithm pseudocode for the proposed average weighted pattern score with attribute rank based feature selection classifier is presented in Fig. 2 .

The attribute selection is a significant step that contributes the classification accuracy. It provides a rank for each attribute in the dataset using mathematical measures. Each attribute will be ranked based on the score obtained by them. The attributes having best rank will be considered as the relevant attributes and least scored attributes are considered as the irrelevant attributes which can be removed. The proposed method for attribute score calculation called attribute score based ranking algorithm ASR algorithm is described. Consider the training dataset $\mathrm{D}$ with $\mathrm{m}$ distinct classes $\mathrm{C}_{\mathrm{i}}$. The overall score of the database based on the number of distinct classes is calculated as in (11).

$$
\operatorname{Score}(D)=\operatorname{Avg}\left(\sum_{i=1}^{m} p_{i}{ }^{p_{i}}\right)
$$

where $\mathrm{p}_{\mathrm{i}}$ is the probability that an arbitrary instance in D that belongs to class $C_{i}$. The formula to calculate $p_{i}$ is shown in (12).

$$
p_{i}=\frac{\text { Number of instances belonging to } C_{i}}{\text { Total number of instances in } D}
$$

To calculate the attribute score for each attribute, having $\mathrm{n}$ distinct values, the count of tuples set having $\mathrm{n}$ distinct values $\left\{\mathrm{n}_{1}, \mathrm{n}_{2}, \mathrm{n}_{3}, \ldots, \mathrm{n}_{\mathrm{j}}\right\}$ are grouped as $\left\{\mathrm{G}_{1}\right.$, $\left.\mathrm{G}_{2}, \ldots, \mathrm{G}_{\mathrm{j}}\right\}$. The calculation of attribute score $\mathrm{A}_{\text {score }}$ is given in (13).

$$
A_{\text {Score }}=\operatorname{Score}(D)-\operatorname{Avg}\left(\sum_{j=1}^{n} p\left(n_{j}\right) X \operatorname{Score}\left(G_{j}\right)\right)
$$

where $p\left(n_{j}\right)$ is the probability that an arbitrary instance in $\mathrm{G}_{\mathrm{j}}$ that belongs to class $\mathrm{C}_{\mathrm{i}}$ 
The score for each attribute is calculated and the rank is evaluated for all the attributes. The attribute score based ranking algorithm is explained in Fig.3.

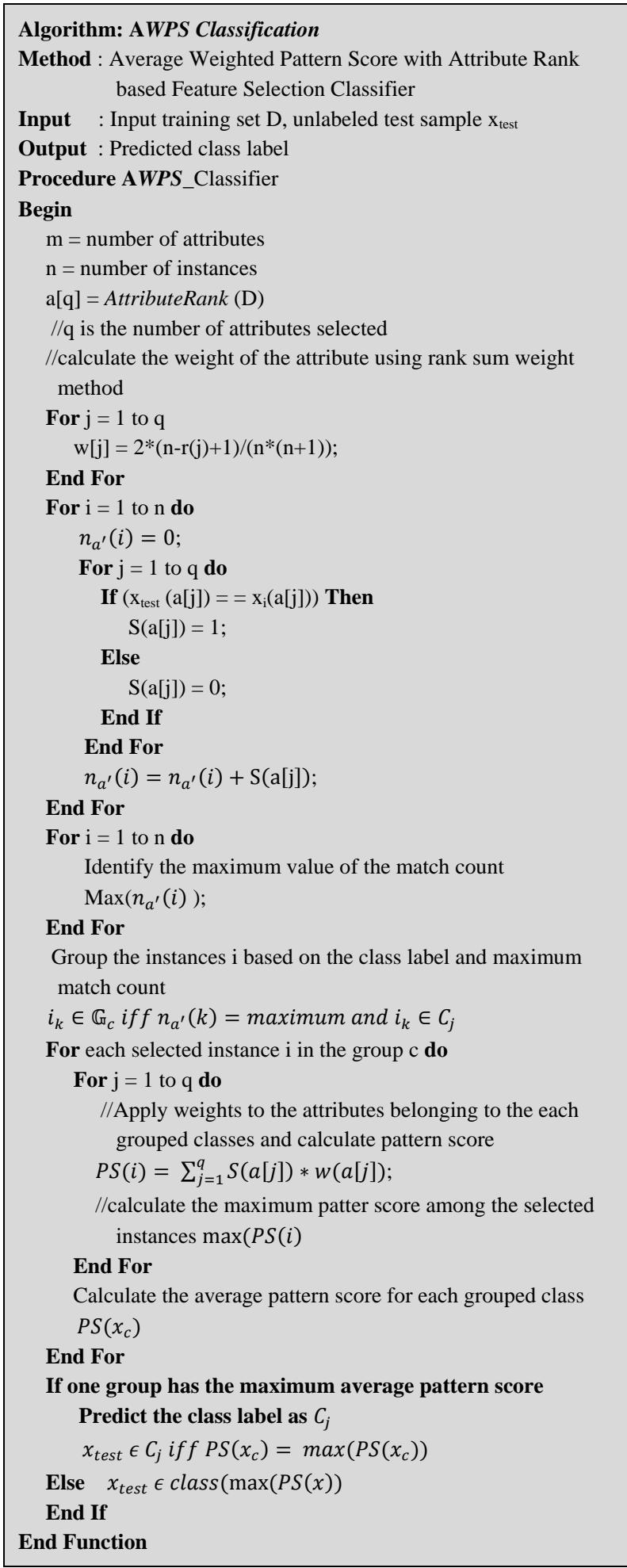

Fig.2. Algorithm pseudocode for the proposed classification method.

To illustrate the attribute score based ranking algorithm, consider the training tuples containing class labels from the AllElectronics Customer Database used by Han et al. (2011) [1] and the records are shown in Table 1. Let $\mathrm{D}$ be the AllElectronics Customer Database having 4 attributes \{age, income, student, credit_rating\} with one class label attribute $\{$ buy_computer $\}$ and 14 training instances.

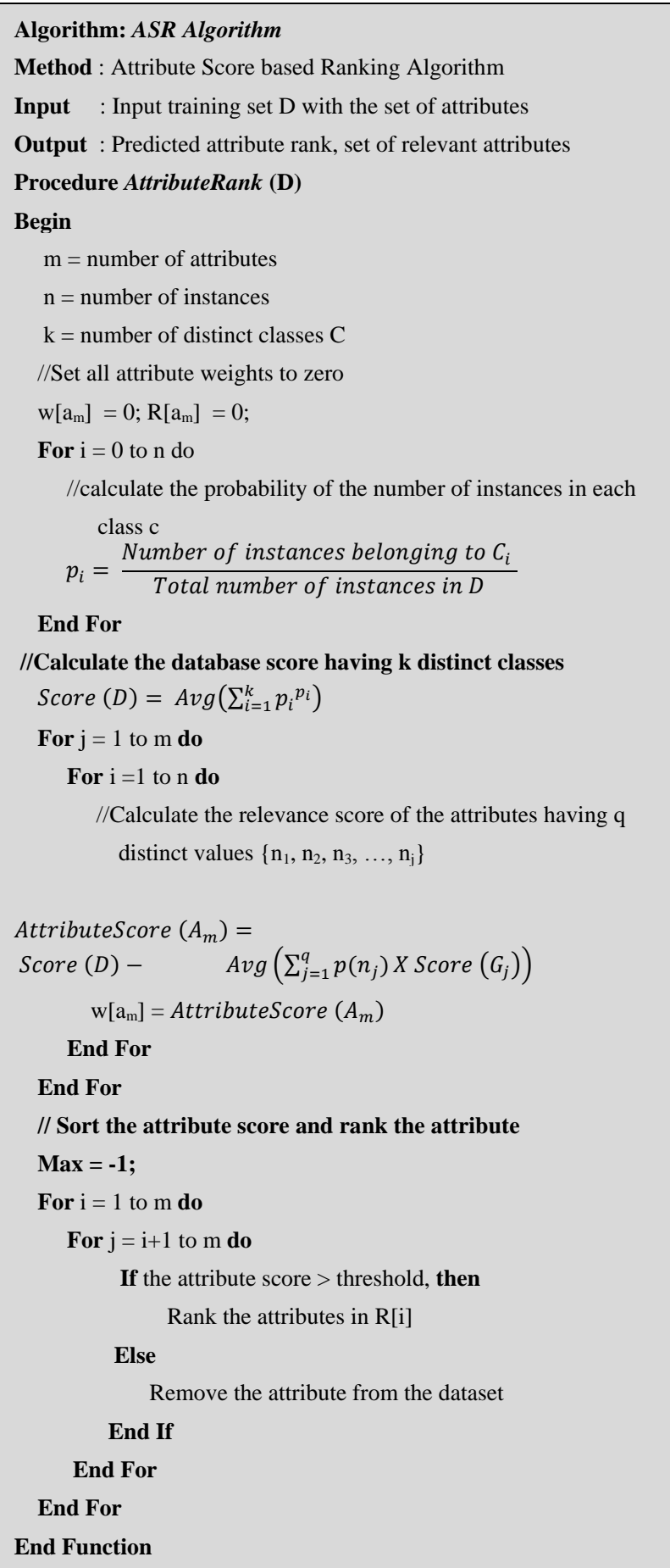

Fig.3. Algorithm Pseudocode for the Attribute Score based Ranking.

Here all the attributes are discrete valued attributes. The number of distinct classes labels of buy_computer attribute is 2 . With $\mathrm{m}$ as 2 the distinct values of the class labels are 'yes' and 'no'. The distinct values of each attribute and the number of instances having these distinct values are depicted in Table 2. 
In Table 2, buy_computer represents the class label attributes in which among 14 instances, 9 instances belong to the 'yes' category and 5 instances belong to the 'no' category.

Table 1. Allelectronics Customer Database

\begin{tabular}{|c|c|c|c|c|c|}
\hline Instance ID & age & income & student & credit_rating & class:buy_computer \\
\hline 1 & youth & high & no & fair & no \\
\hline 2 & youth & high & no & excellent & no \\
\hline 3 & middle_aged & high & no & fair & yes \\
\hline 4 & senior & medium & no & fair & yes \\
\hline 5 & senior & low & yes & fair & yes \\
\hline 6 & senior & low & yes & excellent & no \\
\hline 7 & middle_aged & low & yes & excellent & yes \\
\hline 8 & youth & medium & no & fair & yes \\
\hline 9 & youth & low & yes & fair & yes \\
\hline 10 & senior & medium & yes & fair & yes \\
\hline 11 & youth & medium & yes & excellent & yes \\
\hline 12 & middle_aged & medium & no & excellent & yes \\
\hline 13 & middle_aged & high & yes & fair & no \\
\hline 14 & senior & medium & no & excellent & \\
\hline
\end{tabular}

Table 2. Distinct attribute values with the number of instances in AllElectronics Customer Database

\begin{tabular}{|c|c|}
\hline Attribute Name & Set of Distinct value \\
\hline age & $\{$ youth:5, middle_aged: 4, senior: 5$\}$ \\
\hline income & $\{$ high: 4, medium: 6, low: 4$\}$ \\
\hline student & $\{$ no: 7, yes: 7$\}$ \\
\hline credit_rating & $\{$ fair: 8, excellent: 6$\}$ \\
\hline buy_computer & $\{$ no: 5, yes: 9$\}$ \\
\hline
\end{tabular}

The overall database score is calculated as

$$
\text { Score }(D)=\frac{\left(\left(\frac{9}{14}\right)^{9 / 14}+\left(\frac{5}{14}\right)^{5 / 14}\right)}{2}=\frac{1.446}{2}=0.723 .
$$

Next step is to calculate the attribute score for each attribute in the dataset D. Consider the attribute 'age' having three distinct values \{youth, middle_aged, senior with their corresponding instance count as $\{5,4,5\}$. Grouping the instances in the dataset D based on the attribute's (age) distinct values will result in three groups. The instance ID that belonging to each groups are $\mathrm{G}_{1}$ (youth): $\{1,2,8,9,11\}, \mathrm{G}_{2}$ (middle_aged): $\{3,7,12$, $13\}$ and $\mathrm{G}_{3}$ (senior): $\{4,5,6,10,14\}$. Also in Group $\mathrm{G}_{1}$ (youth), three instances belong to class label 'no' and 2 instances belong to 'yes', in Group $\mathrm{G}_{2}$ (middle_aged), all the 4 instances belong to 'yes' and in $\mathrm{G}_{3}$ (senior), 3 instances belong to 'yes' and 2 belong to 'no'. The details of the attribute 'age' are given in Table 3.

Now the attribute score for age having three distinct values are calculated as follows.

$$
\begin{aligned}
& \text { Score }\left(A_{\text {age }}\right) \\
& =(0.723) \\
& -\frac{5}{14}\left(\frac{\left(\left(\frac{2}{5}\right)^{2 / 5}+\left(\frac{3}{5}\right)^{3 / 5}\right)}{2}\right)+\frac{4}{14}\left(\frac{\left(\left(\frac{4}{4}\right)^{4 / 4}+0\right)}{2}\right)+\frac{5}{14}\left(\frac{\left(\left(\frac{3}{5}\right)^{3 / 5}+\left(\frac{2}{5}\right)^{2 / 5}\right)}{2}\right)
\end{aligned}
$$

$$
\text { Score }\left(A_{\text {age }}\right)=(0.723)-\frac{(0.256)+(0.143)+(0.256)}{3}=0.505
$$

Similarly, the score for other attributes can be calculated.

$$
\begin{gathered}
\operatorname{Score}\left(A_{\text {income }}\right)=0.480 \\
\text { Score }\left(A_{\text {student }}\right)=0.341 \\
\text { Score }\left(A_{\text {credit_rating }}\right)=0.355
\end{gathered}
$$

Thus the attribute age has the highest score with 0.505 and student attribute has the least score with 0.341 . The scores for the attributes income and credit_rating are 0.480 and 0.341 respectively. Once the ranks are identified the weights of each attribute can be calculated using the rank sum method. By using the rank sum weight method, the weights are calculated and are normalized in such a way that the sum of weights of the raked attributes is 1 . The details are shown in Table 4.

Table 3. Attribute details for 'age' attribute in AllElectronics Customer Database

\begin{tabular}{|c|c|c|c|}
\hline \multirow{2}{*}{$\begin{array}{c}\text { Attribute } \\
\text { Value }\end{array}$} & \multicolumn{3}{|c|}{ Instance ID : (Count) } \\
\cline { 2 - 4 } & Overall & $\begin{array}{c}\text { Class } \\
\text { Label : yes }\end{array}$ & $\begin{array}{c}\text { Class } \\
\text { Label : no }\end{array}$ \\
\hline youth & $\{1,2,8,9,11\}:(5)$ & $\{9,11\}:(2)$ & $\{1,2,8\}:(3)$ \\
\hline middle_aged & $\{3,7,12,13\}:(4)$ & $\{3,7,12,13\}:(4)$ & \{\}$:(0)$ \\
\hline senior & $\{4,5,6,10,14\}:(5)$ & $\{4,5,10\}:(3)$ & $\{6,14\}:(2)$ \\
\hline
\end{tabular}

Table 4. Attribute scores, ranks and weights for AllElectronics Customer Database

\begin{tabular}{|c|c|c|c|}
\hline Attribute Name & $\begin{array}{c}\text { Attribute } \\
\text { Score }\end{array}$ & $\begin{array}{c}\text { Attribute } \\
\text { Rank }\end{array}$ & $\begin{array}{c}\text { Rank } \\
\text { Weight }\end{array}$ \\
\hline age & 0.505 & 1 & 0.4 \\
\hline income & 0.480 & 2 & 0.3 \\
\hline student & 0.341 & 4 & 0.1 \\
\hline credit_rating & 0.355 & 3 & 0.2 \\
\hline
\end{tabular}


Table 5. Test samples to be classified

\begin{tabular}{|c|c|c|c|c|}
\hline $\begin{array}{c}\text { Test } \\
\text { sample }\end{array}$ & age & income & student & credit_rating \\
\hline 1 & youth & low & no & excellent \\
\hline 2 & middle_aged & medium & no & fair \\
\hline
\end{tabular}

In this illustration, since, there are only 4 attributes, the attribute 'student' having least score is removed. However, in the case of having numerous attributes, the threshold can be set to filter the relevant attributes. The attributes having scores greater than the threshold values are considered as irrelevant attributes. The working procedure of the proposed weighted pattern matching with attribute rank score based feature selection classifier algorithm is illustrated. For example, consider the test samples to be classified as shown in Table 5 .

The next step is to compare each instance in the test samples with that of the training samples listed in Table 1. The match count and the pattern score of the test sample 1 with respect to the training set are given in table 6 . With respect to the first training instance, the value of the attribute age whose rank weight is 0.4 is the only matching attribute with the first test sample. Thus the corresponding pattern score is 0.4. In the case of the second training instance, the values of attributes age and credit_rating are matched with the first test sample. Thus the pattern score is the sum of the rank weight of age $(0.4)$ and credit_rating (0.2) which is 0.6 . Similarly, the pattern score is calculated for all the training samples. The details are provided in Table 6 .

Table 6. Calculation of match count and pattern score for the test sample 1

\begin{tabular}{|c|c|c|c|c|c|}
\hline age & income & credit_rating & class:buy_computer & match count & Pattern Score \\
\hline youth & high & fair & no & 1 & 0.4 \\
\hline youth & high & excellent & no & 2 & 0.6 \\
\hline middle_aged & high & fair & yes & 0 & 0 \\
\hline senior & medium & fair & yes & 0 & 0 \\
\hline senior & low & fair & yes & 1 & 0.3 \\
\hline senior & low & excellent & no & 2 & 0.5 \\
\hline middle_aged & low & excellent & yes & 2 & 0.5 \\
\hline youth & medium & fair & no & 1 & 0.4 \\
\hline youth & low & fair & yes & 0 & 0.7 \\
\hline senior & medium & fair & yes & 2 & 0 \\
\hline youth & medium & excellent & yes & 1 & 0.6 \\
\hline middle_aged & medium & excellent & yes & 0 & 0.2 \\
\hline middle_aged & high & fair & yes & 1 & 0 \\
\hline senior & medium & excellent & no & & 0.2 \\
\hline
\end{tabular}

The next step is to group the training instances having a maximum match count based on the class label. The average pattern score for each group is calculated. The group having the class label 'no' has the average pattern score as 0.55 and that of the group with a class label 'yes' has the average pattern score as 0.6. Thus the test sample is classified to the class label 'yes' which is having the highest average pattern score of 0.6. The details are given in Table 7.

Table 7. Calculation of the average pattern score for the test sample 1

\begin{tabular}{|c|c|c|c|c|c|c|}
\hline age & income & credit_rating & class:buy_computer & match count & Pattern Score & Average Pattern Score \\
\hline youth & high & excellent & no & 2 & 0.6 & 0.55 \\
\hline senior & low & excellent & no & 2 & 0.5 & 0.5 \\
\hline middle_aged & low & excellent & yes & 2 & 0.7 \\
\hline youth & low & fair & yes & 2 & 0.6 & 0.60 \\
\hline youth & medium & excellent & yes & 2 & 0.6 \\
\hline
\end{tabular}

Table 8. Calculation of average pattern score for the test sample 2

\begin{tabular}{|c|c|c|c|c|c|c|}
\hline age & income & credit_rating & class:buy_computer & match count & pattern score & average pattern score \\
\hline middle_aged & high & fair & yes & 2 & 0.6 & 0.5 \\
\hline Senior & medium & fair & yes & 2 & 0.5 & 0.7 \\
\hline Senior & medium & fair & yes & 2 & 2 & 0.6 \\
\hline middle_aged & medium & excellent & yes & 2 & 0.5 & 0.5 \\
\hline middle_aged & high & fair & yes & 2 & no & 0.5 \\
\hline youth & medium & fair & no & & \\
\hline
\end{tabular}


Table 9. Final predicted class labels for the test samples

\begin{tabular}{|c|c|c|c|c|c|}
\hline Test sample & age & income & student & credit_rating & class:buy_computer \\
\hline 1 & youth & low & yes & Excellent & yes \\
\hline 2 & middle_aged & medium & no & Fair & yes \\
\hline
\end{tabular}

Similarly, the test sample 2 can be classified based on the above process. The average pattern score for the test sample 2 is given in Table 8 . The test sample 2 is classified to the class 'yes' since it is having the highest average pattern score as 0.58 . The final prediction of class labels for the test samples given in table 5 is provided in Table 9.

If the average pattern score is very low for all the groups, then the unlabeled dataset can be predicted as an outlier.

\section{EXPERIMENTAL ANALYSIS}

This section discusses the details about the experimental setup, results, and analysis based on the experiment conducted.

\section{A. Experimental Setup}

The analysis based on the experiment conducted using various datasets has been presented in this section. 40 datasets of various domain available publically at UCI [17] and KEEL [18] repository have been used for the study. The details of the datasets are listed in Table 10. The datasets consist of a different number of instances, attributes, and classes in which nursery dataset has the maximum number of instances as 12960 and lenses dataset has the minimum number of instances as 24 . Similarly, audiology dataset has the maximum number of attributes with the count 69 and Haberman dataset consists of minimum number of attributes as 3 among the datasets used for the study. The number of distinct classes varies from 2 to 24 . As some of the attributes in the datasets have a large number of values, they ridiculously increase the computational complexity. Thus, the values are scaled and normalized between $[0,1]$ using min-max normalization as in Han et al. (2011) [1]. The proposed average weighted pattern score with attribute rank based feature selection (AWPS) algorithm is compared with 11 traditional existing classifiers such as K-Nearest Neighbour classifier (KNN), Decision Tree (TREE), Support Vector Machine (SVM), Random Forest (RF), Neural Network (NN), Naïve Bayes (NB), Logistic Regression (LR), CN2 Rule Inducer (CN), AdaBoost (AB), Data Gravitation Classification (DGC) and Extended Data Gravitation Classification (DGC+).

The proposed algorithm is compared with these 11 existing and most commonly used classifiers and the performance of these algorithms are measured using various measures. Generally, classification accuracy is the most commonly used traditional metric for evaluating the class label prediction. Thus the classification accuracy of the algorithms is measured as in (14).
Table 10. List of Dataset used for the study

\begin{tabular}{|c|c|c|c|c|}
\hline S.No & Dataset & $\begin{array}{c}\text { No. of } \\
\text { Instances }\end{array}$ & $\begin{array}{c}\text { No. of } \\
\text { Attributes }\end{array}$ & $\begin{array}{l}\text { No. of } \\
\text { Classes }\end{array}$ \\
\hline 1 & Appendicitis & 106 & 7 & 2 \\
\hline 2 & audiology & 226 & 69 & 24 \\
\hline 3 & Australian & 690 & 14 & 2 \\
\hline 4 & Balance & 625 & 4 & 3 \\
\hline 5 & Breast cancer & 286 & 9 & 2 \\
\hline 6 & Bupa & 345 & 6 & 2 \\
\hline 7 & Car & 1728 & 6 & 4 \\
\hline 8 & Cardiotocography & 2126 & 23 & 10 \\
\hline 9 & Dermatology & 366 & 33 & 6 \\
\hline 10 & Ecoli & 336 & 7 & 8 \\
\hline 11 & German Credit & 1000 & 20 & 2 \\
\hline 12 & Glass & 214 & 9 & 7 \\
\hline 13 & Haberman & 306 & 3 & 2 \\
\hline 14 & Hayes-Roth & 160 & 4 & 3 \\
\hline 15 & Hepatitis & 155 & 19 & 2 \\
\hline 16 & Ionosphere & 351 & 35 & 2 \\
\hline 17 & Iris & 150 & 4 & 3 \\
\hline 18 & Lenses & 24 & 4 & 3 \\
\hline 19 & Lymphography & 148 & 18 & 4 \\
\hline 20 & Monk-1 & 556 & 7 & 2 \\
\hline 21 & Monk-2 & 301 & 6 & 2 \\
\hline 22 & Monk-3 & 554 & 7 & 2 \\
\hline 23 & Mushroom & 8124 & 22 & 2 \\
\hline 24 & Nursery & 12960 & 8 & 5 \\
\hline 25 & Phoneme & 5404 & 5 & 2 \\
\hline 26 & Pima Diabetes & 768 & 8 & 2 \\
\hline 27 & Solar Flare & 1066 & 11 & 6 \\
\hline 28 & Sonar & 208 & 60 & 2 \\
\hline 29 & Soybean & 683 & 35 & 19 \\
\hline 30 & Spambase & 4597 & 57 & 2 \\
\hline 31 & TAE & 151 & 5 & 3 \\
\hline 32 & Tic-Tac-Toe & 958 & 9 & 2 \\
\hline 33 & Titanic & 2201 & 3 & 2 \\
\hline 34 & Vehicle & 846 & 18 & 4 \\
\hline 35 & Voting & 435 & 16 & 2 \\
\hline 36 & Vowel & 990 & 13 & 11 \\
\hline 37 & WDBC & 569 & 32 & 2 \\
\hline 38 & Wine & 178 & 13 & 3 \\
\hline 39 & Yeast & 1484 & 8 & 10 \\
\hline 40 & Zoo & 101 & 16 & 7 \\
\hline
\end{tabular}

$$
\text { Accuracy }=\frac{\text { Number of Correct Predictions }}{\text { Total Number of Predictions }}
$$

However, classification accuracy alone will not be a correct measure in all the cases. Especially, in case of imbalanced class distribution of the underlying datasets, accuracy does not distinguish the correctly classified 
samples for individual classes. An Area under the ROC Curve (AUC) is one of the good measures for an imbalanced class problem as it deliberates the class distribution for evaluation.

The formula for AUC is presented as

$$
A U C=\frac{1+T P_{\text {rate }}-F P_{\text {rate }}}{2}
$$

Statistical evaluation for the experimental results has been made using Analysis of Variance (ANOVA) which was developed by Ronald Fisher. Generally, it is a statistical hypothesis testing used for testing three or more experimental methods for statistical significance [34]. Thus ANOVA is employed to verify the means of the proposed and existing classifiers are significantly different from each other.

\section{B. Classification Accuracy}

The algorithms are measured and compared using classification accuracy as the primary metrics. The results are detailed in Table 11. The proposed algorithm outperforms well for 19 out of 40 datasets. DGC+ classifier provides better classification rate for 7 out of 40 datasets.

Table 11. Classification Accuracy Comparison

\begin{tabular}{|c|c|c|c|c|c|c|c|c|c|c|c|c|c|}
\hline \multirow{2}{*}{ S.No } & \multirow{2}{*}{ Dataset } & \multicolumn{12}{|c|}{ Classification Algorithms } \\
\hline & & AWPS & DGC + & DGC & $\mathbf{A B}$ & CN2 & LR & NB & $\mathbf{N N}$ & $\mathbf{R F}$ & SVM & TREE & KNN \\
\hline 1 & Appendicitis & 0.898 & 0.841 & 0.871 & 0.811 & 0.792 & 0.858 & 0.719 & 0.868 & 0.858 & 0.868 & 0.792 & 0.868 \\
\hline 2 & udiology & 0.915 & 0.904 & 0.887 & 0.765 & 0.695 & 0.788 & 0.235 & 0.796 & 0.761 & 0.845 & 0.765 & 0.655 \\
\hline 3 & Australian & 0.899 & 0.887 & 0.849 & 0.819 & 0.797 & 0.864 & 0.548 & 0.859 & 0.871 & 0.681 & 0.845 & 0.701 \\
\hline 4 & Balance & 0.904 & 0.899 & 0.899 & 0.760 & 0.795 & 0.922 & 0.914 & 0.982 & 0.824 & 0.922 & 0.794 & 0.718 \\
\hline 5 & Breast cancer & 0.733 & 0.731 & 0.715 & 0.724 & 0.650 & 0.724 & 0.731 & 0.699 & 0.745 & 0.563 & 0.657 & 0.734 \\
\hline 6 & Bupa & 0.668 & 0.674 & 0.653 & 0.661 & 0.649 & 0.681 & 0.678 & 0.645 & 0.655 & 0.580 & 0.626 & 0.675 \\
\hline 7 & Car & 0.995 & 0.952 & 0.913 & 0.977 & 0.950 & 0.879 & 0.863 & 0.994 & 0.946 & 0.845 & 0.972 & 0.845 \\
\hline 8 & Charditography & 0.995 & 0.999 & 0.997 & 0.984 & 0.990 & 0.999 & 0.980 & 0.999 & 0.998 & 0.994 & 0.994 & 0.405 \\
\hline 9 & Dermatology & 0.979 & 0.975 & 0.917 & 0.934 & 0.954 & 0.978 & 0.978 & 0.967 & 0.967 & 0.959 & 0.962 & 0.888 \\
\hline 10 & Ecoli & 0.829 & 0.823 & 0.767 & 0.789 & 0.729 & 0.768 & 0.777 & 0.866 & 0.845 & 0.866 & 0.818 & 0.860 \\
\hline 11 & German Credit & 0.752 & 0.732 & 0.702 & 0.675 & 0.670 & 0.752 & 0.754 & 0.742 & 0.735 & 0.580 & 0.719 & 0.657 \\
\hline 12 & Glass & 0.758 & 0.704 & 0.689 & 0.664 & 0.631 & 0.617 & 0.631 & 0.710 & 0.729 & 0.617 & 0.696 & 0.673 \\
\hline 13 & Haberman & 0.723 & 0.717 & 0.727 & 0.663 & 0.663 & 0.729 & 0.725 & 0.713 & 0.729 & 0.735 & 0.699 & 0.716 \\
\hline 14 & Hayes-Roth & 0.854 & 0.840 & 0.774 & 0.811 & 0.811 & 0.750 & 0.803 & 0.818 & 0.773 & 0.795 & 0.811 & 0.576 \\
\hline 15 & patitis & 0.876 & 0.863 & 0.834 & 0.742 & 0.794 & 0.852 & 0.839 & 0.839 & 0.845 & 0.748 & 0.794 & 0.761 \\
\hline 16 & nosphere & 0.945 & 0.931 & 0.672 & 0.897 & 0.547 & 0.852 & 0.877 & 0.923 & 0.923 & 0.781 & 0.920 & 0.849 \\
\hline 17 & Iris Plants & 0.972 & 0.953 & 0.953 & 0.947 & 0.893 & 0.953 & 0.867 & 0.953 & 0.953 & 0.960 & 0.953 & 0.967 \\
\hline 18 & nses & 0.882 & 0.889 & 0.852 & 0.792 & 0.708 & 0.708 & 0.875 & 0.792 & 0.750 & 0.750 & 0.750 & 0.667 \\
\hline 19 & Lymphography & 0.897 & 0.814 & 0.803 & 0.804 & 0.777 & 0.838 & 0.628 & 0.878 & 0.824 & 0.851 & 0.777 & 0.831 \\
\hline 20 & nk-1 & 0.956 & 0.943 & 0.932 & 0.964 & 0.993 & 0.746 & 0.746 & 0.960 & 0.991 & 0.556 & 0.928 & 0.935 \\
\hline 21 & $\mathrm{kk}-2$ & 0.998 & 0.999 & 0.987 & 0.980 & 0.922 & 0.629 & 0.624 & 0.994 & 0.804 & 0.5 & 0.970 & 0.626 \\
\hline 22 & nk-3 & 0.995 & 0.993 & 0.992 & 0.978 & 0.973 & 0.964 & 0.964 & 0.984 & 0.987 & 0.953 & 0.989 & 0.922 \\
\hline 23 & & 0.999 & 0.998 & 0.987 & 0.945 & 0.999 & 0.987 & 0.955 & 0.986 & 0.965 & 0.9 & 0.985 & 0.965 \\
\hline 24 & irsery & 0.974 & 0.969 & 0.937 & 0.914 & 0.913 & 0.909 & 0.895 & 0.931 & 0.921 & 0.770 & 0.914 & 0.880 \\
\hline 25 & oneme & & 0.871 & 0.847 & 0.873 & 0.792 & 0.750 & 0.749 & 0.847 & 0.897 & 0.465 & 0.864 & 0.880 \\
\hline 26 & $\mathrm{ma}$ & 0.737 & 0.745 & 0.666 & 0.712 & 0.669 & 0.771 & 0.742 & 0.771 & 0.754 & 0.477 & 0.698 & 0.712 \\
\hline 27 & arFlare & & & 0.764 & 0.723 & 0.717 & & 0.628 & 0.722 & 0.725 & & 0.730 & 0.718 \\
\hline 28 & onar & 0.835 & 0.848 & 0.769 & 0.638 & 0.647 & 0.763 & 0.773 & 0.846 & 0.787 & 0.758 & 0.754 & 0.831 \\
\hline 29 & bean & & 0.997 & 0.998 & 0.965 & 0.981 & 0.972 & 0.764 & 0.977 & 0.975 & 0.974 & 0.966 & 0.975 \\
\hline 30 & Spambase & 0.945 & 0.976 & 0.975 & 0.944 & 0.902 & 0.929 & 0.892 & 0.947 & 0.947 & 0.570 & 0.927 & 0.810 \\
\hline 31 & TAE & 0.776 & 671 & 0.670 & 0.762 & 0.789 & 0.636 & 0.689 & 0.742 & 0.768 & 0.715 & 0.775 & 0.603 \\
\hline 32 & Tic-Tac-Toe & 0.894 & 0.854 & 0.690 & 0.941 & 0.892 & 0.951 & 0.694 & 0.945 & 0.953 & 0.788 & 0.943 & 0.796 \\
\hline 33 & & 0.798 & 0.778 & 0.779 & 0.791 & 0.791 & 0.778 & 0.778 & 0.787 & 0.790 & 0.503 & 0.791 & 0.486 \\
\hline 34 & Vehicle & 0.799 & 0.711 & 0.657 & 0.708 & 0.655 & 0.798 & 0.576 & 0.731 & 0.745 & 0.688 & 0.712 & 0.657 \\
\hline 35 & Voting & 0.986 & 0.988 & 0.985 & 0.924 & 0.929 & 0.956 & 0.901 & 0.952 & 0.954 & 0.949 & 0.949 & 0.926 \\
\hline 36 & Vowel & 0.985 & 0.982 & 0.979 & 0.831 & 0.775 & 0.649 & 0.597 & 0.979 & 0.928 & 0.914 & 0.803 & 0.958 \\
\hline 37 & WDBC & 0.975 & 0.989 & 0.975 & 0.912 & 0.923 & 0.951 & 0.947 & 0.977 & 0.944 & 0.968 & 0.924 & 0.926 \\
\hline 38 & Wine & 0.972 & 0.973 & 0.970 & 0.697 & 0.854 & 0.949 & 0.966 & 0.978 & 0.966 & 0.955 & 0.860 & 0.713 \\
\hline 39 & Yeast & 0.598 & 0.593 & 0.515 & 0.557 & 0.555 & 0.589 & 0.589 & 0.595 & 0.573 & 0.595 & 0.541 & 0.589 \\
\hline 40 & Zoo & 0.945 & 0.955 & 0.935 & 0.960 & 0.970 & 0.960 & 0.921 & 0.950 & 0.960 & 0.960 & 0.911 & 0.931 \\
\hline \multicolumn{2}{|c|}{ Average Accuracy } & 0.881 & 0.868 & 0.837 & 0.823 & 0.803 & 0.823 & 0.770 & 0.866 & 0.852 & 0.767 & 0.832 & 0.772 \\
\hline \multicolumn{2}{|c|}{ Average Rank } & 2.625 & 3.875 & 6.350 & 7.600 & 8.225 & 5.975 & 8.425 & 4.250 & 4.925 & 8.050 & 7.300 & 8.375 \\
\hline
\end{tabular}




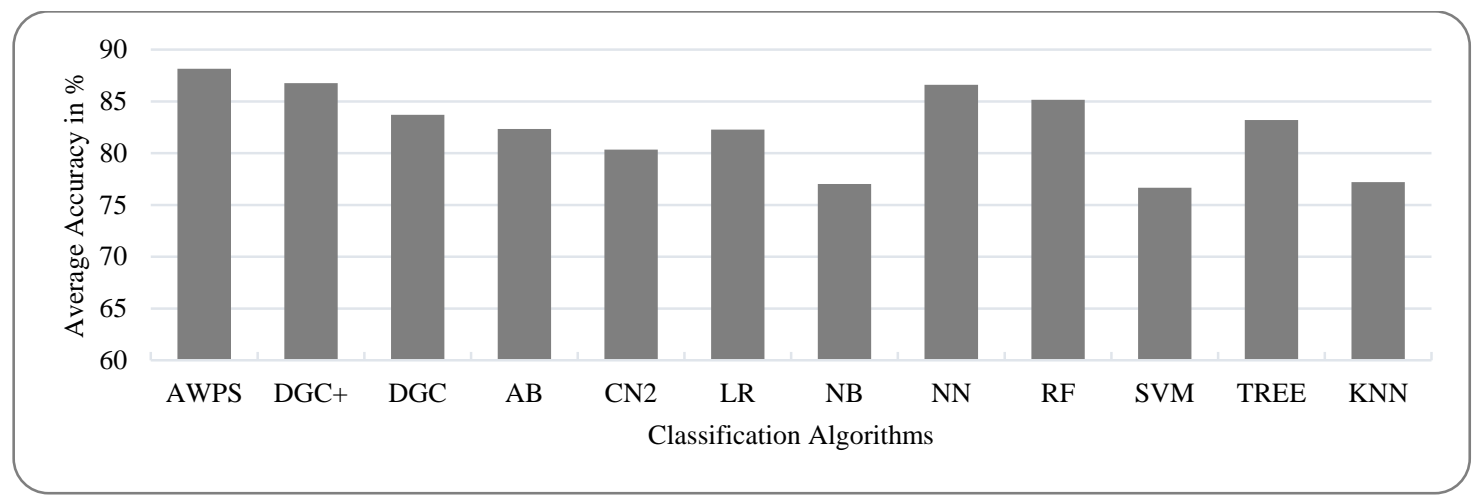

Fig.4. Average classification accuracy.

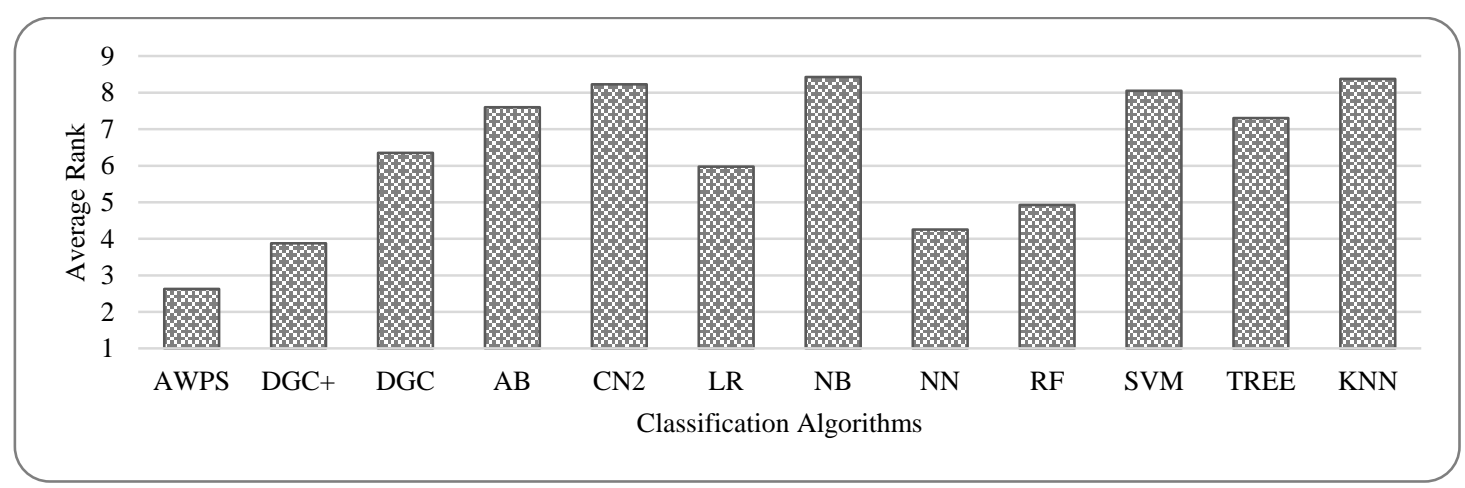

Fig.5. Average rank of the classification algorithms for accuracy.

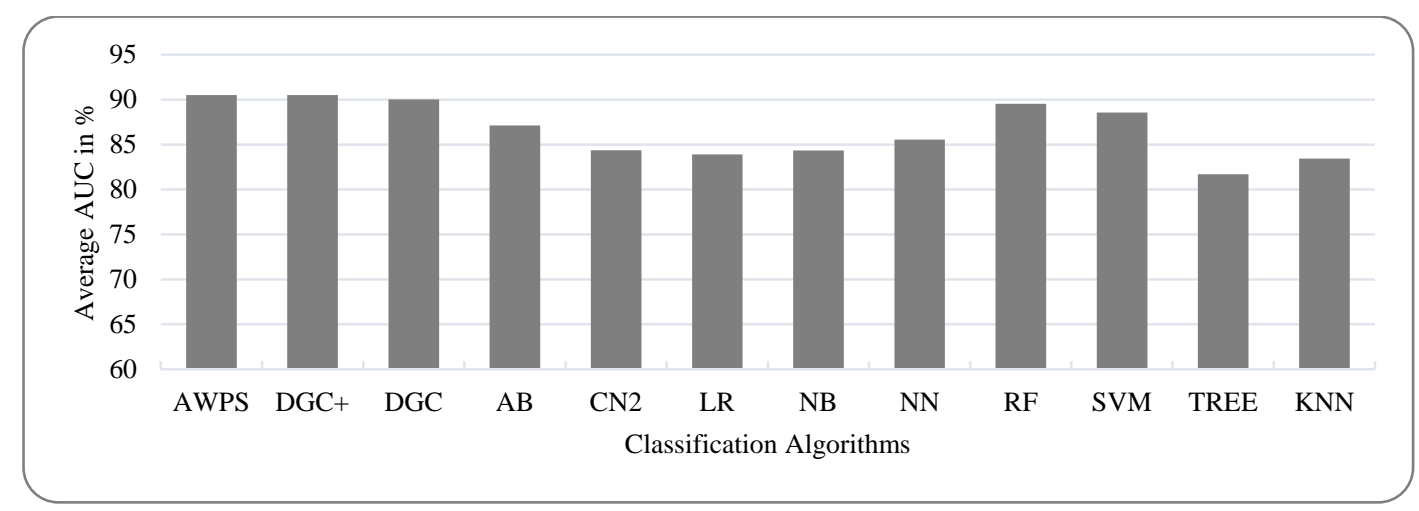

Fig.6. Average AUC values.

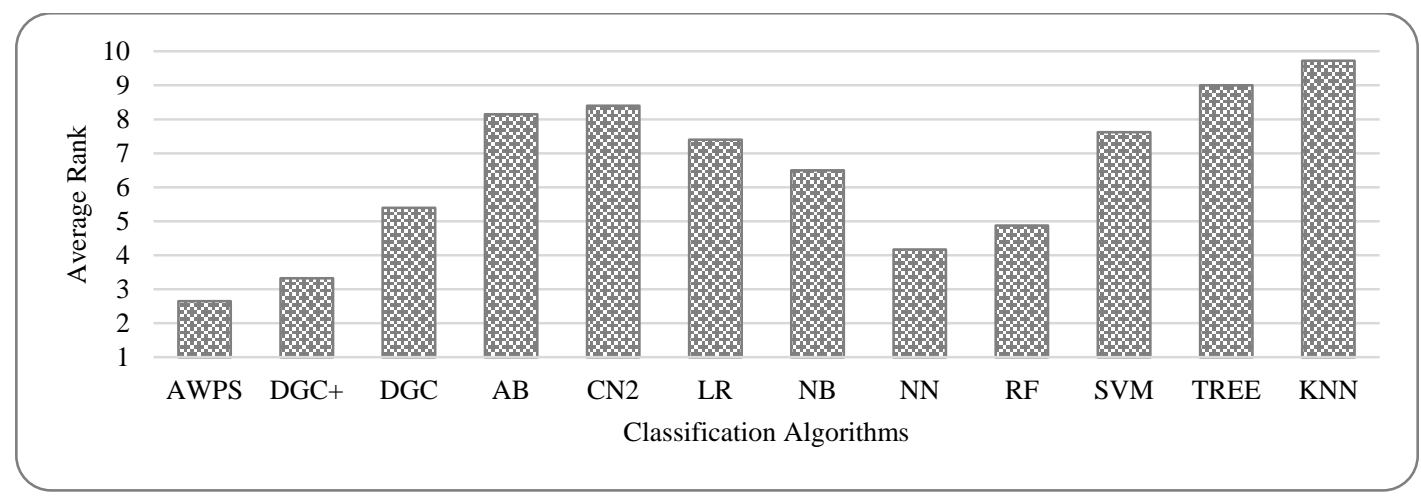

Fig.7. Average rank of the classification algorithms for AUC 
Table 12. Classification AUC Comparison

\begin{tabular}{|c|c|c|c|c|c|c|c|c|c|c|c|c|c|}
\hline \multirow{2}{*}{ S.No } & \multirow{2}{*}{ Dataset } & \multicolumn{12}{|c|}{ Classification Algorithms } \\
\hline & & AWPS & DGC+ & DGC & AB & CN2 & LR & NB & NN & $\mathbf{R F}$ & SVM & TREE & KNN \\
\hline 1 & Appendicitis & 0.884 & 0.854 & 0.835 & 0.697 & 0.814 & 0.797 & 0.833 & 0.869 & 0.786 & .830 & 0.714 & 0.798 \\
\hline 2 & Audiology & 0.892 & 0.889 & 0.875 & 0.846 & 0.869 & 0.857 & 0.832 & 0.850 & 0.855 & 0.823 & 0.874 & 0.807 \\
\hline 3 & Australian & 0.899 & 0.886 & 0.852 & 0.817 & 0.883 & 0.903 & .878 & 0.934 & 0.936 & 0.713 & 0.812 & 0.744 \\
\hline 4 & Balance & 0.862 & 0.875 & 0.805 & 0.799 & 0.805 & 0.827 & 0.842 & 0.887 & 0.877 & 0.889 & 0.816 & 0.783 \\
\hline 5 & Breast cancer & 0.645 & 0.647 & 0.608 & 0.666 & 0.597 & 0.597 & 0.612 & 0.684 & 0.604 & 0.550 & 0.587 & 0.600 \\
\hline 6 & Bupa & 0.699 & 0.674 & 0.653 & 0.650 & 0.647 & 0.625 & 0.651 & 0.669 & 0.610 & 0.602 & 0.625 & 0.612 \\
\hline 7 & Car & 0.994 & 0.998 & 0.991 & 0.973 & 0.908 & 0.927 & 0.971 & 0.981 & 0.993 & 0.949 & 0.897 & 0.945 \\
\hline 8 & Charditography & 0.999 & 0.996 & 0.997 & 0.992 & 0.919 & 0.945 & 0.987 & 0.985 & 0.987 & 0.991 & 0.904 & 0.844 \\
\hline 9 & Dermatology & 0.989 & 0.991 & 0.917 & 0.958 & 0.932 & 0.906 & 0.845 & 0.990 & 0.992 & 0.987 & 0.874 & 0.847 \\
\hline 10 & Ecoli & 0.978 & 0.957 & 0.951 & 0.856 & 0.909 & 0.922 & 0.958 & 0.963 & 0.957 & 0.962 & 0.884 & 0.945 \\
\hline 11 & German Credit & 0.751 & 0.743 & 0.702 & 0.619 & 0.672 & 0.708 & 0.765 & 0.749 & 0.758 & 0.578 & 0.650 & 0.575 \\
\hline 12 & Glass & 0.865 & 0.854 & 0.759 & 0.773 & 0.814 & 0.797 & 0.851 & 0.853 & 0.818 & 0.815 & 0.807 & 0.798 \\
\hline 13 & Haberman & 0.698 & 0.689 & 0.687 & 0.608 & 0.593 & 0.560 & 0.690 & 0.678 & 0.669 & 0.559 & 0.615 & 0.599 \\
\hline 14 & Hayes-Roth & 0.936 & 0.948 & 0.961 & 0.914 & 0.904 & 0.895 & 0.952 & 0.946 & 0.927 & 0.949 & 0.902 & 0.834 \\
\hline 15 & Hepatitis & 0.771 & 0.763 & 0.734 & 0.641 & 0.782 & 0.715 & 0.775 & 0.760 & 0.750 & 0.716 & 0.677 & 0.584 \\
\hline 16 & Ionosphere & 0.950 & 0.931 & 0.672 & 0.890 & 0.714 & 0.882 & 0.927 & 0.960 & 0.968 & 0.811 & 0.836 & 0.917 \\
\hline 17 & Iris Plants & 0.999 & 0.994 & 0.995 & 0.960 & 0.887 & 0.957 & 0.979 & 0.987 & 0.986 & 0.989 & 0.878 & 0.897 \\
\hline 18 & Lenses & 0.891 & 0.897 & 0.871 & 0.824 & 0.798 & 0.848 & 0.857 & 0.851 & 0.882 & 0.874 & 0.841 & 0.802 \\
\hline 19 & Lymphography & 0.925 & 0.923 & 0.935 & 0.811 & 0.877 & 0.804 & 0.887 & 0.945 & 0.930 & 0.908 & 0.793 & 0.904 \\
\hline 20 & Monk-1 & 0.972 & 0.956 & 0.945 & 0.988 & 0.899 & 0.716 & 0.729 & 0.965 & 0.979 & 0.579 & 0.920 & 0.894 \\
\hline 21 & $\mathrm{nk}-2$ & 0.970 & 0.978 & 0.974 & 0.890 & 0.928 & 0.536 & 0.534 & 0.975 & 0.874 & 0.492 & 0.981 & 0.678 \\
\hline 22 & nk-3 & 0.991 & 0.988 & 0.989 & 0.954 & 0.945 & 0.988 & 0.984 & 0.982 & 0.981 & 0.978 & 0.969 & 0.977 \\
\hline 23 & Mushroom & 0.999 & 0.995 & 0.996 & 0.974 & 0.921 & 0.994 & 0.991 & 0.995 & 0.994 & 0.992 & 0.935 & 0.927 \\
\hline 24 & Irsery & 0.984 & 0.979 & 0.937 & 0.985 & 0.983 & 0.983 & 0.979 & 0.984 & 0.983 & 0.927 & 0.978 & 0.979 \\
\hline 25 & honeme & 0.875 & 0.866 & 0.851 & 0.848 & 0.819 & 0.812 & 0.828 & 0.896 & 0.889 & 0.479 & 0.811 & 0.879 \\
\hline 26 & ima & 0.788 & 0.765 & 0.741 & 0.689 & 0.740 & 0.726 & 0.719 & 0.734 & 0.781 & 0.587 & 0.616 & 0.738 \\
\hline 27 & SolarFlare & 0.912 & 0.916 & 0.929 & 0.898 & 0.917 & 0.939 & 0.917 & 0.928 & 0.921 & 0.915 & 0.898 & 0.899 \\
\hline 28 & nar & 0.886 & 0.891 & 0.811 & 0.633 & 0.692 & 0.789 & 0.856 & 0.879 & 0.885 & 0.799 & 0.721 & 0.882 \\
\hline 29 & bean & & 0.998 & 0.997 & 0.948 & 0.884 & 0.957 & 0.968 & 0.945 & 0.849 & 0.988 & 0.966 & 0.844 \\
\hline 30 & Spambase & 0.960 & 0.979 & 0.980 & 0.975 & 0.949 & 0.961 & 0.960 & 0.980 & 0.982 & 0.709 & 0.922 & 0.877 \\
\hline 31 & & 0.788 & 0.784 & 0.765 & 0.748 & 0.755 & 0.635 & 0.642 & 0.704 & 0.798 & 0.639 & 0.785 & 0.619 \\
\hline 32 & Tic-Tac-Toe & 0.878 & 0.865 & 0.712 & 0.832 & 0.818 & 0.852 & 0.748 & 0.849 & 0.876 & 0.864 & 0.856 & 0.765 \\
\hline 33 & Titanic & & 0.769 & 0.772 & 0.768 & 0.768 & 0.759 & 0.716 & 0.768 & 0.766 & 0.500 & 0.768 & 0.656 \\
\hline 34 & Vehicle & 0.871 & 0.857 & 0.827 & 0.805 & 0.838 & 0.843 & 0.809 & 0.860 & 0.817 & 0.895 & 0.800 & 0.799 \\
\hline 35 & Voting & 0.990 & 0.987 & 0.978 & 0.933 & 0.963 & 0.995 & 0.972 & 0.992 & 0.991 & 0.979 & 0.880 & 0.912 \\
\hline 36 & Vowel & 0.985 & 0.982 & 0.979 & 0.907 & 0.897 & 0.946 & 0.946 & 0.911 & 0.981 & 0.975 & 0.856 & 0.811 \\
\hline 37 & WDBC & 0.989 & 0.987 & 0.975 & 0.930 & 0.859 & 0.905 & 0.971 & 0.975 & 0.986 & 0.980 & 0.902 & 0.888 \\
\hline 38 & Wine & 0.965 & 0.973 & 0.970 & 0.824 & 0.834 & 0.965 & 0.991 & 0.987 & 0.948 & 0.955 & 0.889 & 0.875 \\
\hline 39 & Yeast & 0.996 & 0.991 & 0.987 & 0.972 & 0.851 & 0.978 & 0.885 & 0.987 & 0.879 & 0.954 & 0.971 & 0.960 \\
\hline 40 & Zoo & 0.981 & 0.998 & 0.935 & 0.954 & 0.979 & 0.989 & 0.981 & 0.979 & 0.980 & 0.999 & 0.964 & 0.845 \\
\hline \multicolumn{2}{|c|}{ Average AUC } & 0.905 & 0.900 & 0.871 & 0.844 & 0.839 & 0.844 & 0.855 & 0.895 & 0.886 & 0.817 & 0.834 & 0.813 \\
\hline \multicolumn{2}{|c|}{ Average Rank } & 2.650 & 3.325 & 5.400 & 8.150 & 8.400 & 7.400 & 6.500 & 4.175 & 4.875 & 7.625 & 9.000 & 9.725 \\
\hline
\end{tabular}

The average accuracy of the proposed algorithm is higher than other existing algorithms. The average accuracy of AWPS is $88.1 \%$ and that of DGC+ is $86.8 \%$. Neural Networks is having equally high performance as DGC+. Ensemble classifiers such as random forest, support vector machine and Adaboost provides better results. The average accuracy is depicted in Fig. 4.

The proposed algorithm provides the first rank for 19 datasets. It takes the first and the second positions for 24 datasets and the top three positions for 29 datasets. The average rank of each algorithm employed in the experimental study for the classification accuracy has been computed. The average rank for the proposed algorithm AWPS is 2.625. The average ranks of DGC+, neural network, and random forest are 3.875, 4.25 and 4.925 respectively. The graph for the average rank is depicted in Fig.5.

The statistical analysis has been carried out for the classification accuracy using ANOVA. The statistical model creates $\mathrm{F}$-distribution with $\mathrm{F}$ value $=3.867$ and $\mathrm{F}$ critical value $=1.809$. Thus with the critical difference of 2.508 , the results are significant at a 5\% significance level. The null hypothesis has been rejected by concluding that there is a difference between the classification accuracy of the algorithms under study.

The performances of the classification algorithms used 
in the study are measured using another metric AUC which is significant for imbalanced class data [35]. The values of AUC for all the algorithm and for each dataset are calculated and are shown in Table 12. The proposed algorithm shows the maximum value for 17 out of 40 datasets. DGC + classifier and neural networks provide better AUC value.

The average AUC value for the proposed AWPS algorithm is $90.5 \%$. The average AUC values for the DGC+, neural networks, and random forest are approximately $90 \%$ which are considered as the better performance. However, AWPS perform well and provide a better result than the other algorithms. The average values of AUC for the classification algorithms are depicted in Fig.6.

The proposed algorithm provides the best result for 17 datasets. It takes the top two positions for 23 datasets and the second position for 24 datasets and top three positions for 27 datasets. The average rank of each algorithm employed in the experimental study for the AUC has been computed. The average rank for the proposed algorithm AWPS is 2.65. The average ranks of DGC+, neural network, and random forest are 3.325, 4.175 and 4.875 respectively. The graph for the average rank of AUC is depicted in Fig.7.

Table 13. Evaluation Time Comparison

\begin{tabular}{|c|c|c|c|c|c|c|}
\hline Dataset & AWPS (ms) & DGC+ $(\mathrm{ms})$ & DGC (ms) & NN (ms) & RF (ms) & SVM (ms) \\
\hline Appendicitis & 1.6245 & 5.1123 & 3.5426 & 9.5918 & 10.3895 & 7.7856 \\
\hline Audiology & 2.5863 & 5.2811 & 2.2255 & 9.7611 & 10.5514 & 8.1425 \\
\hline Australian & 4.5449 & 8.5507 & 6.3932 & 13.0287 & 13.9218 & 11.4216 \\
\hline Balance & 4.4541 & 5.7123 & 3.8936 & 10.1921 & 10.8475 & 8.5124 \\
\hline Breast cancer & 1.9666 & 5.6986 & 3.7708 & 10.1781 & 10.6742 & 8.3462 \\
\hline Bupa & 2.3324 & 5.6432 & 3.7519 & 10.1237 & 10.8214 & 8.4212 \\
\hline Car & 5.0563 & 11.8909 & 10.1174 & 16.3707 & 17.5123 & 14.7564 \\
\hline Charditography & 6.1256 & 14.6403 & 10.4586 & 19.1174 & 19.8921 & 17.5114 \\
\hline Dermatology & 2.1236 & 9.9809 & 8.1417 & 14.4605 & 15.1473 & 12.7621 \\
\hline Ecoli & 2.0870 & 5.7185 & 4.1192 & 10.1981 & 10.1589 & 8.5779 \\
\hline German Credit & 4.1135 & 13.1787 & 9.3940 & 17.6583 & 18.9563 & 15.8754 \\
\hline Glass & 2.0154 & 5.9765 & 4.2315 & 10.4552 & 11.1289 & 8.5478 \\
\hline Haberman & 1.9885 & 5.1919 & 3.4609 & 9.6705 & 10.1596 & 7.8579 \\
\hline Hayes-Roth & 1.9631 & 5.0787 & 3.4939 & 9.5572 & 10.3578 & 7.5124 \\
\hline Hepatitis & 1.9253 & 5.4077 & 3.7607 & 9.8882 & 10.8476 & 8.2415 \\
\hline Ionosphere & 3.9431 & 10.5558 & 7.8677 & 15.0289 & 15.9467 & 13.3247 \\
\hline Iris Plants & 1.8662 & 5.1143 & 3.4446 & 9.5932 & 10.4758 & 7.9912 \\
\hline Lenses & 1.4988 & 4.8908 & 3.1446 & 9.3689 & 10.2478 & 7.6452 \\
\hline Lymphography & 1.8315 & 5.2809 & 3.5715 & 9.7587 & 10.6472 & 8.0132 \\
\hline Monk-1 & 2.8125 & 5.9141 & 4.2473 & 10.3936 & 11.1425 & 8.6523 \\
\hline Monk-2 & 1.7642 & 6.6457 & 4.4919 & 11.1247 & 11.8932 & 9.4124 \\
\hline Monk-3 & 1.7479 & 5.9226 & 4.2308 & 10.4021 & 11.1348 & 8.4123 \\
\hline Mushroom & 13.8399 & 26.0123 & 19.4919 & 30.4921 & 31.2617 & 28.7856 \\
\hline Nursery & 21.0258 & 114.0142 & 69.1257 & 118.4937 & 119.2163 & 116.7523 \\
\hline Phoneme & 9.4252 & 23.0148 & 17.4606 & 27.4942 & 28.2987 & 25.7123 \\
\hline Pima & 2.4446 & 10.2452 & 7.7213 & 14.7246 & 15.6124 & 13.4719 \\
\hline SolarFlare & 4.6582 & 13.8917 & 10.6717 & 18.3709 & 19.2478 & 16.7752 \\
\hline Sonar & 2.3467 & 12.7983 & 11.2292 & 17.2777 & 18.1793 & 15.5443 \\
\hline Soybean & 2.3178 & 6.9121 & 4.6919 & 11.5913 & 12.3972 & 9.6635 \\
\hline Spambase & 10.8023 & 56.9142 & 42.4606 & 61.3930 & 62.1863 & 59.6846 \\
\hline TAE & 1.7723 & 5.2453 & 3.4934 & 9.7244 & 10.6732 & 8.1247 \\
\hline Tic-Tac-Toe & 2.2632 & 9.5250 & 8.8306 & 14.1143 & 14.9875 & 12.3689 \\
\hline Titanic & 6.1236 & 25.2518 & 19.4917 & 29.7317 & 30.5288 & 28.1459 \\
\hline Vehicle & 2.1815 & 12.3209 & 9.7250 & 16.9145 & 17.7349 & 15.1475 \\
\hline Voting & 1.5567 & 5.6910 & 3.9754 & 10.1723 & 10.8752 & 8.2514 \\
\hline Vowel & 1.9256 & 13.0810 & 9.4224 & 17.5647 & 18.7236 & 15.2278 \\
\hline WDBC & 2.1201 & 6.2451 & 4.2473 & 10.6243 & 11.7963 & 9.2345 \\
\hline Wine & 1.8523 & 6.3743 & 4.3708 & 10.7581 & 11.9896 & 9.1856 \\
\hline Yeast & 4.8734 & 14.2447 & 9.1424 & 18.3257 & 19.4712 & 17.2256 \\
\hline Zoo & 2.9828 & 5.6626 & 2.8718 & 10.2435 & 10.2578 & 9.0789 \\
\hline Mean Evaluation Time & 3.8721 & 13.1208 & 9.2544 & 17.5983 & 18.4073 & 15.9026 \\
\hline
\end{tabular}




\section{Time Performance}

Apart from accuracy and AUC, the evaluation time of the proposed method is also compared with the existing techniques. The computational complexity using Big-O notation for the proposed algorithm is $\mathrm{O}(\mathrm{nm})$ where $n$ is the number of records in the datasets and $\mathrm{m}$ is the number of attributes selected using proposed attribute score based ranking algorithm. The computation complexity of the other existing algorithms having better classification accuracy or AUC values such as DGC, DGC+, RF, SVM and $\mathrm{NN}$ are $\mathrm{O}\left(\mathrm{mn}^{2}\right), \mathrm{O}\left(\mathrm{mn}^{2}\right), \mathrm{O}(\mathrm{tmn}(\log \mathrm{n})), \mathrm{O}\left(\mathrm{n}^{3}\right)$, and $\mathrm{O}\left(\mathrm{n}^{4}\right)$ where $\mathrm{n}$ is the number of records and $\mathrm{m}$ is the number of attributes. The computational complexity of DGC and DGC+ are same and the complexity of the RF algorithm also depends on the number of trees to be constructed denoted as t. Thus, the proposed algorithm has less computational complexity and thus it is considered faster than other algorithms.

The evaluation time to classify an unlabeled sample using the proposed AWPS algorithm is compared with that of other algorithms such as DGC+, DGC, NN, RF and SVM using the 40 datasets listed in Table 10. The valuation time for all the mentioned algorithms are measured and the values are shown in Table 13. The proposed method has less evaluation time for all the datasets than DGC+, NN, RF and SVM. However, the execution time of AWPS algorithm is less than the DGC algorithm for all the datasets except Audiology, Balance, and Zoo. Also the mean Evaluation time for the proposed AWPS algorithm is $3.87 \mathrm{~ms}$, and for the existing algorithms such as DGC+ is $13.12 \mathrm{~ms}$, DGC is $9.25 \mathrm{~ms}$, $\mathrm{NN}$ is $17.6 \mathrm{~ms}, \mathrm{RF}$ is $18.41 \mathrm{~ms}$ and SVM is $15.9 \mathrm{~ms}$. Thus it is found that the proposed AWPS method has less computational time for classifying the datasets.

\section{Statistical Analysis and Discussion}

The statistical analysis has been carried out for the AUC values of all the methods using ANOVA. The statistical model creates F-distribution with $\mathrm{F}$ value $=$ 2.957 and $\mathrm{F}$ critical value $=1.809$. Thus with the critical difference of 1.148 , the results are significant at a $5 \%$ significance level. The null hypothesis has been rejected by concluding that there is a difference between the AUC value of the algorithms under study.

On average, the datasets used for the experimental analysis consist of 1312 instances, 16.33 attributes, and 4.5 classes, out of which the proposed algorithm provides better performance in classification accuracy and acquire top 3 positions for 29 among 40 datasets having the average of 1403 instances, 17.3 attributes, and 4.6 classes.

Similarly, the proposed algorithm has a good AUC value than other algorithms and obtains top 3 positions for 27 datasets having mean values as 1412 instances, 16.6 attributes, and 5.15 classes. DGC+, neural networks and ensemble methods provide better accuracy. However, AWPS algorithm provide even better results than other algorithms.

\section{CONCLUSION}

In this paper, an average weighted pattern score with attribute rank based feature selection classifier has been suggested for classifying the datasets in an improved way. The proposed feature selection algorithm computes the attribute scores based on their contribution towards better classification. An average weighted pattern score based classification algorithm is suggested for better classification of unlabeled datasets. The main advantage of the proposed AWPS is that its simplicity and better performance than many existing classification methods. The proposed method attained better classification accuracy and AUC values for most of the imbalanced datasets under study when compared with other existing classifiers. The AWPS classification algorithm is well suitable to deal with imbalanced class problem. Statistical analysis were also performed to validate the results obtained from the experiments to support the high performance of the proposal. It is also shown that the evaluation time for the proposed classifier is lower than other significant existing classification algorithms.

\section{REFERENCES}

[1] J. Han, J. Pei and M. Kamber, Data mining: concepts and techniques. Elsevier, 2011.

[2] I. H. Witten, E. Frank, M. A. Hall and C. J. Pal, Data Mining: Practical machine learning tools and techniques. Morgan Kaufmann, 2016.

[3] I. Kononenko and M. Kukar, "Machine Learning and Data Mining: Introduction to Principles and Algorithms," Cambridge, U.K.: Horwood Publ, 2007.

[4] G. V. Lashkia and L. Anthony, "Relevant, irredundant feature selection and noisy example elimination," IEEE Trans. Syst., Man, Cybern. B, Cybern., vol. 34, no. 2, pp. 888-897, Apr. 2004.

[5] M. Paliwal and U. A. Kumar, "Neural networks and statistical techniques: A review of applications," Expert Syst. Appl., vol. 36, no. 1, pp. 2-17, Jun. 2009.

[6] G. Lin, C. Shen, Q. Shi, A. Van den Hengel and D. Suter, "Fast supervised hashing with decision trees for highdimensional data," in Proc. of IEEE Conference on Computer Vision and Pattern Recognition, pp. 1963-1970, 2014.

[7] T. R. Patil and S. S. Sherekar, "Performance analysis of Naive Bayes and J48 classification algorithm for data classification," International journal of computer science and applications, 6(2), pp. 256-261, 2013

[8] D. W. Aha, D. Kibler, and M. K. Albert, "Instance-based learning algorithms," Mach. Learn., vol. 6, no. 1, pp. 3766, Jan. 1991.

[9] M. Muja. and D. G. Lowe, "Scalable nearest neighbor algorithms for high dimensional data," IEEE Transactions on Pattern Analysis \& Machine Intelligence, pp. 22272240, 2014.

[10] I. Naseem, R. Togneri. and M. Bennamoun, "Linear regression for face recognition," IEEE transactions on pattern analysis and machine intelligence, 32(11), pp. 2106-2112, 2010.

[11] A. Y. $\mathrm{Ng}$ and M. I. Jordan, "On discriminative vs. generative classifiers: A comparison of logistic regression and naive bayes," Advances in neural information processing systems, pp. 841-848, 2002. 
[12] P. G. Espejo, S. Ventura, and F. Herrera, "A survey on the application of genetic programming to classification," IEEE Trans. Syst., Man, Cybern. C, Appl. Rev., vol. 40, no. 2, pp. 121-144, Mar. 2010.

[13] T. G. Dietterich, "Ensemble methods in machine learning," International workshop on multiple classifier systems, Springer, Berlin, Heidelberg, pp. 1-15, June 2000.

[14] A. Liaw and M. Wiener, "Classification and regression by random Forest”, R news, 2(3), pp.18-22, 2002.

[15] T. K. An and M. H. Kim, "A new diverse AdaBoost classifier," in Proc. of Artificial Intelligence and Computational Intelligence (AICI), IEEE, vol. 1, pp. 359363, 2010.

[16] S. W. Lin, Z. J. Lee, S. C. Chen and T. Y. Tseng, "Parameter determination of support vector machines and feature selection using simulated annealing approach," Appl. Soft Comput. 8, pp. 1505-1512, 2008.

[17] A. Frank and A. Asuncion, "UCI machine learning repository", Univ. California, School Inf. Comput. Sci., Irvine, CA. [Online]. Available: http://archive.ics.uci.edu/ml/citation_policy.html

[18] J. Alcalá-Fdez, A. Fernandez, J. Luengo, J. Derrac, S. García, L. Sánchez and F. Herrera, "KEEL data-mining software tool: Data set repository, integration of algorithms and experimental analysis framework," J. Multiple-Valued Logic Soft Comput., vol. 17, pp. 255287, 2011.

[19] J. V. Tu, "Advantages and disadvantages of using artificial neural networks versus logistic regression for predicting medical outcomes," Journal of clinical epidemiology, 49(11), pp. 1225-1231, 1996.

[20] S. Dreiseitl and L. Ohno-Machado, "Logistic regression and artificial neural network classification models: a methodology review", Journal of biomedical informatics, 35(5-6), pp. 352-359, 2002.

[21] S. S. Nikam, "A comparative study of classification techniques in data mining algorithms," Oriental Journal of Computer Science \& Technology, 8(1), pp.13-19, 2015.

[22] D. L. Poole and A K. Mackworth, "Artificial Intelligence: foundations of computational agents," Cambridge University Press, 2010.

[23] S. J. Press and S. Wilson, "Choosing between logistic regression and discriminant analysis," Journal of the American Statistical Association, 73(364), pp. 699-705, 1978, 1978.

[24] Leo Breiman, "Random Forests," in Machine Learning, 45(1), pp. 5-32, 2001.

[25] J. R. Quinlan, "Bagging, Boosting, and C4.5," in Proc. of national conference on artificial intelligence, pp. 725-730.

[26] S. C. Chen, S. W. Lin, S. Y. Chou, "Enhancing the classification accuracy by scatter-search-based ensemble approach," Appl. Soft Comput. 11, pp. 1021-1028, 2011.

[27] K. Ming Leung, "K-Nearest Neighbor Algorithm for Classification," Polytechnic University Department of Computer Science/Finance and Risk Engineering, 2007.

[28] B. Li, Y. W. Chen, and Y. Q. Chen, "The nearest neighbor algorithm of local probability centers," IEEE Trans. Syst., Man, Cybern. B, Cybern., vol. 38, no. 1, pp. 141-154, Feb. 2008.

[29] L. Peng, B. Peng, Y. Chen, A. Abraham, "Data gravitation based classification," Inf.Sci. 179, pp. 809819, march 2009.

[30] J. Wang, P. Neskovic, and L. N. Cooper, "Improving nearest neighbor rule with a simple adaptive distance measure," Pattern Recognit. Lett., vol. 28, no. 2, pp. 207 213, Jan. 2007.
[31] Y. Zong-Chang, "A vector gravitational force model for classification,” Pattern Anal. Appl., 11, pp.169-177, May 2008.

[32] A. Cano, A. Zafra, S. Ventura, "Weighted data gravitation classification for standard and imbalanced data," IEEE Trans. Cybern. 43, December 2013.

[33] N.K. Sreeja, and A. Sankar, "Pattern matching based classification using ant colony optimization based feature selection," Applied Soft Computing, 31, pp.91-102, 2015.

[34] G. Hesamian, "One-way ANOVA based on interval information," International Journal of Systems Science, 47(11), pp .2682-2690, 2016

[35] V. López, A. Fernandez, S. Garcia, V. Palade and F. Herrera, "An Insight into Classification with Imbalanced Data: Empirical Results and Current Trends on Using Data Intrinsic Characteristics," Information Sciences, 250, pp. 113-141, 2013

\section{Authors' Profiles}

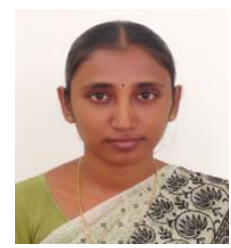

Sathya Bama Subramani completed her Doctor of Philosophy in Computer Applications under Anna University Chennai, Tamil Nadu, India. Her area of interest includes Data mining, Web mining, Computer Networks and Database. She has an experience of 10 years in teaching and 7 years in research with a good number of publications.

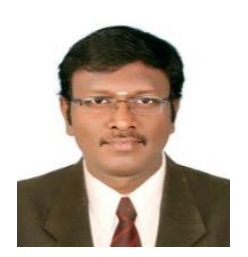

Saravanan Arumugam completed his Doctor of Philosophy in Computer Applications under Anna University Chennai, Tamil Nadu, India. He is currently working as a Director and Professor in the Department of Computer Science and Applications, Sree Saraswathi Thyagaraja College, Pollachi, Coimbatore Tamil Nadu, India. He has an experience of 20 years in teaching and 9 years in research with a good number of publications. His area of interest includes Web Security, Network Security, Web Mining, Software Engineering and Database.

How to cite this paper: S. Sathya Bama, A. Saravanan, "Efficient Classification using Average Weighted Pattern Score with Attribute Rank based Feature Selection", International Journal of Intelligent Systems and Applications(IJISA), Vol.11, No.7, pp.29-42, 2019. DOI: 10.5815/ijisa.2019.07.04 